J. Laub

Nagoya Math. J.

Vol. 83 (1981), 1-4

\title{
A SINGULAR CONVOLUTION KERNEL WITHOUT PSEUDO-PERIODS
}

\author{
JESPER LAUB
}

Let $G$ be a locally compact abelian group and $N$ a non-zero convolution kernel on $G$ satisfying the domination principle. We define the cone of $N$-excessive measures $E(N)$ to be the set of positive measures $\xi$ for which $N$ satisfies the relative domination principle with respect to $\xi$. For $\xi \in E(N)$ and $\Omega \subseteq G$ open the reduced measure of $\xi$ over $\Omega$ is defined as

$$
R_{\xi}^{\rho}=\inf \{\eta \in E(N) \mid \eta \geq \xi \text { in } \Omega\} .
$$

Further discussion of excessive and reduced measures is given in [4] and [5].

Let $\vartheta$ denote the set of compact neighbourhoods of $O$, the neutral element of $G$. The convolution kernel $N$ is said to be singular if

$$
R_{N}^{\& V}=N \text { for all } V \in \vartheta .
$$

$A$ point $x \in G$ is called a pseudo-period of $N$ if there exists a number $c>0$ such that

$$
N * \varepsilon_{x}=c N,
$$

where $\varepsilon_{x}$ denotes the Dirac-measure at $x$. The set of pseudo-periods of $N$ is a closed subgroup of $G$.

In [3] Itô gave the following result (Corollaire 2):

$A$ convolution kernel $N$ satisfying the domination principle is singular if and only if the group of pseudo-periods of $N$ is non-compact.

The "if" part of the statement is easy to prove (cf. e.g. [1]), but the "only if" statement is false in general, although it seems reasonable due to obvious examples. It is our purpose to give a counterexample to this statement.

Suppose that there exists a strictly decreasing sequence $\left(G_{n}\right)_{n \in N}$ of closed non-compact subgroups of $G$ 


$$
G=G_{1} \supset G_{2} \supset G_{3} \supset \cdots
$$

satisfying $\bigcap_{n=1}^{\infty} G_{n}=\{0\}$. We denote by $\omega_{G_{n}}$ a Haar-measure on $G_{n}$. Let $\varphi$ be a fixed non-zero positive continuous function with compact support and put $a_{n}=\sup _{x \in G} \omega_{G_{n}} * \varphi(x), n \in N$.

The convolution kernel, which we will consider, is

$$
\kappa=\sum_{n=1}^{\infty} \frac{1}{2^{n} a_{n}} \omega_{G_{n}} .
$$

Since every positive continuous function with compact support can be majorized by a finite linear combination of translates of $\varphi$, it follows that the series converges vaguely. Furthermore $\kappa$ is shift-bounded.

$1^{\circ}$. The only pseudoperiod of $\kappa$ is 0 .

Since $\kappa$ is shift-bounded, we have $c=1$ for a pseudo-period $x \in G$ of $\kappa$. If $x \neq 0$, then we can find $i \in N$ such that $x \in G_{i} \mid G_{i+1}$ and therefore

$$
\begin{aligned}
\kappa * \varepsilon_{x} & =\sum_{n=1}^{i} \frac{1}{2^{n} a_{n}} \omega_{G_{n}}+\sum_{n=i+1}^{\infty} \frac{1}{2^{n} a_{n}} \omega_{G_{n}} * \varepsilon_{x} \\
\kappa & =\sum_{n=1}^{i} \frac{1}{2^{n} a_{n}} \omega_{G_{n}}+\sum_{n=i+1}^{\infty} \frac{1}{2^{n} a_{n}} \omega_{G_{n}} .
\end{aligned}
$$

These two expressions cannot be equal, since $x$ belongs to the support of the second term of $\kappa * \varepsilon_{x}$, but not to support of the second term of $\kappa$.

$2^{\circ}$. $\kappa$ satisfies the domination principle.

We shall need the following two lemmas, which are both easily proved

LemMa 1 (Itô [2]). Let $N$ be a shift-bounded convolution kernel and $\omega_{G}$ a Haar-measure on $G$. If $N$ satisfies the domination principle, then $N+\omega_{G}$ satisfies the domination principle.

Lemma 2. Let $N$ be a convolution kernel on $G$ and $H$ a closed subgroup of $G$ such that $\operatorname{supp} N \subseteq H$. Then $N$ satisfies the domination principle as convolution kernel on $G$ if and only if $N$ satisfies the domination principle as convolution kernel on $H$.

By repeated use of these lemmas it follows, that the partial sum

$$
\kappa_{k}=\sum_{n=1}^{k} \frac{1}{2^{n} a_{n}} \omega_{G_{n}}, \quad k \in N
$$

satisfies the domination principle. Since the set of convolution kernels satisfying the domination principle is vaguely closed and $\kappa=\lim _{k \rightarrow \infty} \kappa_{k}$, we 
have that $\kappa$ satisfies the domination principle.

$3^{\circ}$. $\kappa$ is singular.

Let $V \in \vartheta$ be given and choose for $i \in N$ a point $x_{i} \in G_{i} \mid G_{i+1}$ such that $x_{i} \notin V-\operatorname{supp} \varphi$. Then we have

$$
\begin{aligned}
\kappa * \varepsilon_{x_{i}} * \varphi= & \sum_{n=1}^{i} \frac{1}{2^{n} a_{n}} \omega_{G_{n}} * \varphi+\sum_{n=i+1}^{\infty} \frac{1}{2^{n} a_{n}} \varepsilon_{x_{i}} * \omega_{G_{n}} * \varphi \\
& \leq R_{\kappa * \varphi}^{\varphi V V}+2^{-i} \text { in } \mathscr{C} V
\end{aligned}
$$

However since $\operatorname{supp}\left(\varepsilon_{x_{i}} * \varphi\right) \subseteq \mathscr{C} V$ and $R_{\kappa * \varphi}^{\mathscr{V}}+2^{-i} \in E(\kappa)$ we obtain

$$
\sum_{n=1}^{i} \frac{1}{2^{n} a_{n}} \omega_{G_{n}} * \varphi \leq \kappa * \varepsilon_{x_{i}} * \varphi \leq R_{\kappa * \varphi}^{\varphi V}+2^{-i},
$$

and by letting $i$ tend to infinity we get $R_{\kappa * \varphi}^{\varphi v}=\kappa * \varphi$. Finally Lemma 1.8 in [5] gives

$$
\kappa * \varphi=\lim _{V \uparrow G} R_{s * \varphi}^{\& V}=\left(\lim _{V \uparrow G} R_{x}^{\& V}\right) * \varphi
$$

which shows that $R_{\kappa}^{\varphi V}=\kappa$ for all $V \in \vartheta$.

ExAmple. For $G=Z, G_{n}=2^{n-1} Z=\left\{2^{n-1} k \mid k \in Z\right\}$ and $\varphi$ the function which takes the value 1 at 0 and 0 elsewhere we get

$$
\kappa(\{0\})=1 ; \kappa(\{m\})=1-2^{-i-1}, m \neq 0
$$

where $i$ is the largest non-negative integer for which $2^{i}$ divides $m$.

Remark. If a singular convolution kernel $N$ satisfies the balayage principle for all open sets, then the group of pseudo-periods of $N$ is noncompact, because if $\varepsilon_{\mathscr{E} V}^{\prime}$ denotes a $N$-balayaged measure of $\varepsilon_{0}$ on $\mathscr{C} V, V \in \vartheta$, then we have $N=N * \varepsilon_{\mathscr{G} V}^{\prime}$. Consequently $N$ has a pseudo-period in supp $\varepsilon_{\mathscr{\mathscr { C }} V}^{\prime} \subseteq \overline{\mathscr{C} V}$ by Proposition 7 in [3].

\section{REFERENCES}

[1] Berg, C. and Laub, J., The resolvent for a convolution kernel satisfying the domination principle, Preprint Series 1977 No. 41, Dept. of Math., Univ. of Copenhagen.

[2] Itô, M., Une caractérisation du principe de domination pour les noyaux de convolution, Japan J. Math., New series 1, No. 1 (1975), 5-35.

[ 3 ] — Caractérisation du principe de domination pour les noyaux de convolution non-bornés, Nagoya Math., J., 57 (1975), 167-197.

[4] - Sur le principe relatif de domination pour les noyaux de convolution, Hiroshima Math. J., 5 (1975), 293-350. 
[5] Laub, J., On unicity of the Riesz decomposition of an excessive measure, Math. Scand., to appear.

\section{Matematisk Institut}

Universitetsparken 5

DK-2100 Kobenhavn $\phi$

Denmark 